\title{
Treatment strategies for management of MCA-MI spontaneous dissecting aneurysms
}

\begin{abstract}
Perforators along the middle cerebral artery (MCA) M1 territory increase the difficulty of treating dissecting aneurysms. Such lesions may only require observation or complex staged procedures requiring augmentation techniques which reverse flow from the dissecting segment while preserving parent vessel lumen and distal perfusion. A neurovascular registry containing nearly 900 patient admissions from October 2008 through February 2013 comprised 18 patients with fusiform MCA-M1 aneurysms; their records were analyzed to assess demographics, presenting symptoms, co-morbidities, treatments, and outcomes. Patient ages ranged from 22 to 73 years, averaging at 56 . The population was predominately female $(69 \%)$. Unruptured aneurysm was the most frequent presentation $(69 \%)$, followed by subarachnoid hemorrhage (22\%), and stroke $(11 \%)$. Subarachnoid hemorrhage patients were Hunt Hess grade III $(\mathrm{n}=1)$ and IV $(\mathrm{n}=1)$. Co-morbidities included hypertension $(77 \%)$, smoking $(62 \%)$, and hyperlipidaemia $(46 \%)$. Two patients $(15 \%)$ experienced regrowth after initial treatment requiring further intervention. Treatment was most often surgical $(85 \%)$ and varied among four categories: observation only; simple surgical, including occlusion without parent vessel compromise; transitional, characterized by flow reversal using proximal occlusion or trapping; and maximal combined, defined as flow reversal with bypass to augment MCA distal circulation. Aneurysm management included wraps (47\%), bypass with stent, clip or occlusion (27\%), coil and stent (13\%), bypass with coil (7\%), and observation $(7 \%)$. Seven treatments $(47 \%)$ were staged procedures. Post-procedure complications included hydrocephalus $(27 \%)$, vasospasm (20\%), and seizures $(20 \%)$. The 30-day mortality rate was $15 \%(\mathrm{n}=2)$. The sample size precludes statistical analysis the relationship between treatment type and complications or mortality.
\end{abstract}

Keywords: Bypass, Cerebral revascularization, Fusiform aneurysm, Middle cerebral artery, Dissecting aneurysms
Volume I Issue 4 - 2014

\author{
Eric S Nussbaum, ' Archie Defillo, ${ }^{2}$ Mushtaq \\ H Qureshi, ${ }^{2}$ Andrea Zelensky ${ }^{3}$ \\ 'Department of Neurosurgery, John Nasseff Neuroscience \\ Institute, USA \\ ${ }^{2}$ Department of Neurosurgery Centracare Health System St. \\ Cloud, USA \\ ${ }^{3}$ Health East Care Systems, St Joseph's Hospital, USA
}

Correspondence: Archie Defillo, Clinical Director Neurosciences, St. Cloud Hospital Centracare Health, USA, Tel |-320-25 |-2700, Fax |-320-25|-0000,

Email defilloa@centracare.com

Received: August 14, 2014 | Published: August 18, 2014
Abbreviations: ACA, Anterior Cerebral Artery; CT, Computed Tomography; EC-IC, Extra Cranial-Intra Cranial; ICA, Internal Carotid Artery; MCA, Middle Cerebral Artery; SAH, Subarachnoid Hemorrhage; STA-MCA, Superficial Temporal Artery to Middle Cerebral Artery; ICG, IndoCyanine Green

\section{Introduction}

The majority of MCA aneurysms are saccular lesions that arise at the MCA bifurcation region. ${ }^{1-5}$ Aneurysms involving the M1 segment are less common, accounting for approximately $12 \%$ of MCA aneurysms, and are generally small saccular lesions at the origin of the anterior temporal artery or a lenticulostriatal perforator. ${ }^{6,7}$ MCA-M1 fusiform lesions vary significantly from their saccular counterparts. ${ }^{1,8-10}$

Spontaneous MCA-M1 fusiform aneurysms are unusual lesions. Non atherosclerotic dissection has been associated with congenital vascular processes including Moyamoya disease, local fenestration, Fibromuscular dysplasia, alpha-1antitrypsin deficiency, disorders of collagen/elastin, infections, polyarteritis nodosa, and neoplastic invasion of the intracranial arterial wall. ., $, 811,12-15^{-15}$

Fusiform intracranial aneurysms can be grouped into different categories based on clinical presentation. Patients presenting with an acute SAH tend to be younger with unstable aneurysms and management challenges. Patients presenting with ischemic injury and those identified incidentally appear to be older with larger, more atheromatous or calcified lesions.

Perhaps due to the relative rarity of MCA-M1 fusiform lesions, there is limited literature available. ${ }^{10}$ The following series describes our experiences various treatment modalities in the management of these challenging lesions.

\section{Material and methods}

A neurovascular registry containing nearly 900 admissions from October 2008 through February 2013 was used to identify 18 patients with non-traumatic, spontaneous, fusiform MCA-M1 aneurysms. Patient records were analyzed to assess patient's demographics, presenting symptoms, co-morbidities, and aneurysm characteristic. Neuroimaging investigations included CT-scans, MRI when available, and angiograms. Selection of treatments was reviewed for single, combined, staged and related in-hospital, 30 day clinical outcomes. Additional cases treated at our center prior to instituting the registry were also included for review.

\section{Results}

Medical records of the 18 revealed the average patient age was 52 years and ranged from 22 to 73 years. The population was predominately female $(69 \%)$. The majority of aneurysms were unruptured (69\%), with subarachnoid hemorrhage occurring in $22 \%$ and stroke in $11 \%$. Common co-morbidities included smoking $(62 \%)$, hypertension (77\%), and hyperlipidaemia (46\%).

Treatment was most often surgical (85\%) Aneurysm management included wrapping with or without a clip (40\%), bypass with stent, clip or occlusion (25\%), clip (10\%), coil and stent $(10 \%)$, bypass $(5 \%)$, bypass with coil $(5 \%)$, and observation $(5 \%)$. Five patients $(28 \%)$ underwent staged procedures. Three patients $(17 \%)$ experienced re growth after initial treatment and required further intervention. 
Post-procedure complications included anemia (30\%), hydrocephalus (20\%) and vasospasm (15\%). The 30-day mortality rate was $11 \%(n=2)$. The small sample size precludes statistical analysis of how type of treatment may relate to complications or mortality rates.

\section{Discussion}

Fusiform intracranial aneurysms most often affect the Vertebrobasilar system with severe atherosclerosis playing a significant role. ${ }^{1-5,6,7}$ Day et al. ${ }^{1}$ analyzed 40 patients with fusiform aneurysms involving the MCA treated over two decades, and added another 62 patients identified from the English literature that had been published between 1950 and 2000. In their description, $80 \%$ of the lesions presented with non-hemorrhagic symptoms or were discovered incidentally. In our series, $67 \%$ of cases presented as asymptomatic unruptured aneurysms. Additional clinical presentations included subarachnoid hemorrhage (22\%) and stroke (11\%). In Ohkuma's report of symptomatic non-traumatic MCA dissecting aneurysms, $69.2 \%$ of patients presented with $\mathrm{SAH}$ and $30.8 \%$ with cerebral ischemia. ${ }^{10}$ The higher percentage of SAH may be related to selection bias.

SAH appears to affect younger patients and presents unique management challenges. Endovascular therapy with stenting or coils requires antiplatelet therapy which may be problematic after acute SAH. Surgical exposure of a recently ruptured dissecting aneurysm carries the potential for severe and uncontrolled intra-operative rupture of a lesion that may not be readily amenable to simple clip reconstruction. In this setting, distal bypass combined with proximal or distal M1 occlusion may represent reasonable options to reverse or limit flow through the abnormal arterial segment ${ }^{7,9,16-20}$ In three of our cases, this paradigm only temporarily stabilized the situation, and further treatment was required to treat an enlarging aneurysm.

\section{Surgical strategies}

A variety of surgical techniques were used to treat these complex lesions. Aneurysms discovered incidentally without evidence of progressive enlargement were often managed with surgical exploration to assess for possible clip reconstruction. When surgical exposure demonstrated multiple perforating vessels emerging from the aneurysm itself, simple wrapping with clips used to secure the wrapped segment was performed. ${ }^{21}$

In the setting of SAH or in the management of giant or enlarging aneurysms producing mass effect, endovascular therapy was chosen when feasible or, more commonly, some form of distal revascularization combined with either surgical or endovascular parent artery occlusion. A low-flow bypass technique (superficial temporal artery-middle cerebral artery anastomosis) was the treatment of choice. In three of these patients, a staged approach was employed. In the remainder, bypass was followed by parent artery occlusion at the same setting. Overall, revascularization was utilized in $39 \%$ of our patients.

Endovascular management of dissecting aneurysms consists of coil embolization, with or without additional stent assisted techniques. Parent artery occlusion can be utilized only if adequate collateral circulation can be confirmed. Our 30-day mortality rate was $11 \%$ for patients presenting with acute dissection. In a series of 4,000 aneurysms described by Drake, the incidence of giant fusiform lesions was $3 \%$. Of those, $21 \%$ of aneurysms were located in the anterior circulation, and only $0.33 \%$ were considered MCA giant fusiform. Overall, good outcomes were achieved in only $8(62 \%)$ of the 13 MCA patients. The direct-operative morbidity rate, including a single death from an unruptured aneurysm was $23 \%$. Drake et al described 55 patients who developed giant fusiform aneurysms of the MCA, and whom were treated with a variety of adjunct interventions, wrapping technique being one of them. In his series wrapping failed to arrest aneurysm growth in one patient; concomitant EC-IC bypass as rescue therapy was needed for a second patient, and a third patient was left hemiplegic after complete suture encasement of the aneurysm was performed with mersaline. ${ }^{8}$

\section{Illustrative case descriptions}

Case 1: A previously healthy 22-year old woman presented as a HuntHess and Fisher grade II SAH (Figure 1A). Her initial angiogram confirmed a $2 \mathrm{~cm}$ right MCA-M1 dissecting aneurysm involving the mid to distal M1 trunk. This aneurysm extended into the bifurcation and proximal M2 origin region (Figure 1B). The lesion was associated with $50 \%$ stenosis of the M1 proximal segment (Figure 1C). Her initial treatment consisted of stent assisted coil embolization (Figure 1D). Post procedure she developed cerebral vasospasm and delayed hydrocephalus, managed with lumbar drainage. Seventeen days later she was discharged home without neurological deficit. Two months post-discharge she presented with acute onset left sided weakness (Figure 1E). Readmission diagnostic angiogram showed significant interval aneurysm growth. Of interest, most of the lenticulostriatal vessels arose from an anatomical variant in which an accessory anterior division right MCA formed from the right ACA A1/ A2 junction (Figure 1F).

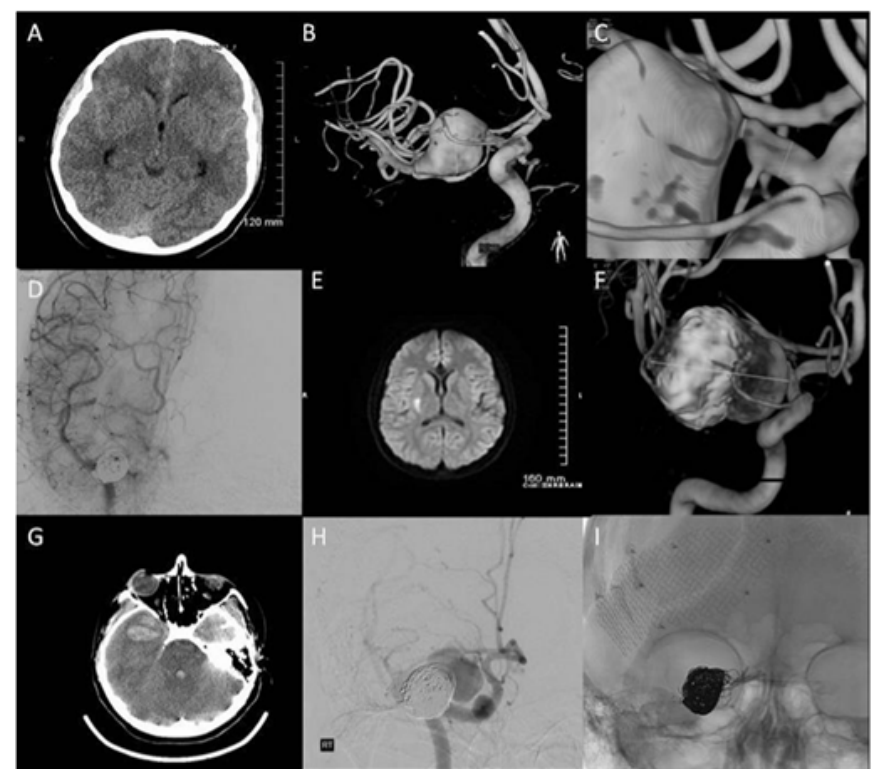

Figure I IA) Admission CT-scan demonstrating a grade II Fisher SAH; IB) 3D angiogram reconstruction showing a $2 \mathrm{~cm}$ right $\mathrm{MCA}-\mathrm{MI}$ dissecting aneurysm involving the mid to distal MI trunk; IC) 3D image highlighting significant inflow stenosis ( $\geq 50 \%$ ) of the proximal MI trunk; ID) Near complete occlusion of the dissecting MCA-MI aneurysm after stent assisted coil embolization; IE) Confirmed right basal ganglia infarct on the TI-weighted MRI following acute neurological symptoms 2 months after her initial endovascular treatment; IF) Readmission angiogram showing a significant interval aneurysm growth and the anatomical relationship of the perforator vessels to the lesion; IG) CTscan performed after a second acute neurological deterioration demonstrating a $3 \mathrm{~cm}$ right temporal lobe intraparenchymal hematoma, combined with right to left shift and uncal herniation; IH) pre-operative emergency catheter angiography showing additional growth of the dissecting aneurysm, this lesion was now associated with no perceivable flow in the distal MI and proximal M2 segments; II) intra-operative angiogram demonstrating occlusion of the aneurysm after permanent clip occlusion of the right supraclinoid ICA. 
That evening, the patient suffered a new SAH with acute respiratory arrest. A repeat CT-scan revealed a $3 \mathrm{~cm}$ right temporal lobe hematoma with intraventricular extension, right to left midline shift, and uncal herniation (Figure 1G). Emergency catheter angiography demonstrated additional growth of the dissecting aneurysm with no perceivable flow in the distal M1 and proximal M2 segments (Figure $1 \mathrm{H})$.

The patient underwent an emergency decompressive craniectomy. Upon opening the dura, the aneurysm ruptured spontaneously causing active arterial bleeding through the temporal lobe. The microscope was brought into the field, the frontal lobe was retracted, the supraclinoid internal carotid (ICA) was exposed, and a temporary clip was applied. A persistent hemorrhage continued, presumably resulting from collateral supply from the opposite side via the A1 segment. Temporary clipping of the A1 segment stopped the bleeding, allowing careful dissection and placement of a permanent clip across the M1 origin. Remarkably, removal of the temporary clip from the ICA resulted in bleeding from the ICA itself, raising the possibility of an underlying arteriopathy as the vessels seemed friable. Two permanent clips were placed along the injured supraclinoid ICA. Angiography showed good cross filling of both A2 vessels as well as the accessory MCA. As the ICA and MCA were occluded, there was no further filling of the aneurysm (Figure 1I).

A temporal tip lobectomy was performed to augment decompressive craniectomy to decompress the 3 rd cranial nerve and brain stem structures. Given the patient's young age, a decision was made to perform a superficial temporal artery to middle cerebral artery bypass using standard revascularization technique. Excellent flow through the anastomosis was confirmed by intra-operative indocyanine green (ICG) angiography with good filling of the distal MCA branches. After a prolonged ICU stay, the patient was discharged to a rehabilitation facility for active care. At the 18 month followup, the patient was ambulating with an ankle brace, had a residual left-sided hemiparesis, was fully interactive but had modest cognitive dysfunction and disinhibition.

Case 2: A 43 year-old man with undiagnosed systemic hypertension presented as a Hunt Hess grade III and Fisher grade IV SAH (Figure $2 \mathrm{~A}$ and $2 \mathrm{~B}$ ). His admission MRI and angiogram revealed a $15 \mathrm{~mm}$ fusiform left M1 segment aneurysm. Additionally, there was a small, early anterior frontal branch arising directly from the body of the fusiform dilatation, as well as the presence of lateral lenticulostriatal perforator vessels (Figure 2C and 2D). This was associated with sluggish filling into the distal MCA beyond the aneurysm (Figure $2 \mathrm{E}$ ). Incorporation of collateral vessels within the aneurysm complex precluded endovascular intervention. Proposed management consisted of microsurgical exploration with attempted EC-IC bypass and M1 distal occlusion.

\section{Operative description}

The surgical procedure began with a standard STA-MCA bypass;intra-operative indocyanine green video angiography confirmed bypass patency. The distal M1 portion beyond the aneurysm was identified, and a temporary clip was placed. An intra-operative angiogram confirmed good filling of the distal circulation through the bypass and native collateral flow. The temporary clip was then replaced with a permanent clip. Subsequent angiography revealed good flow through the bypass into the distal MCA circulation, with near complete occlusion of the fusiform lesion (Figure 2F). Postoperative MRI demonstrated several areas of ischemia on the left MCA territory. At discharge, the patient had a Glasgow outcome scale of two.

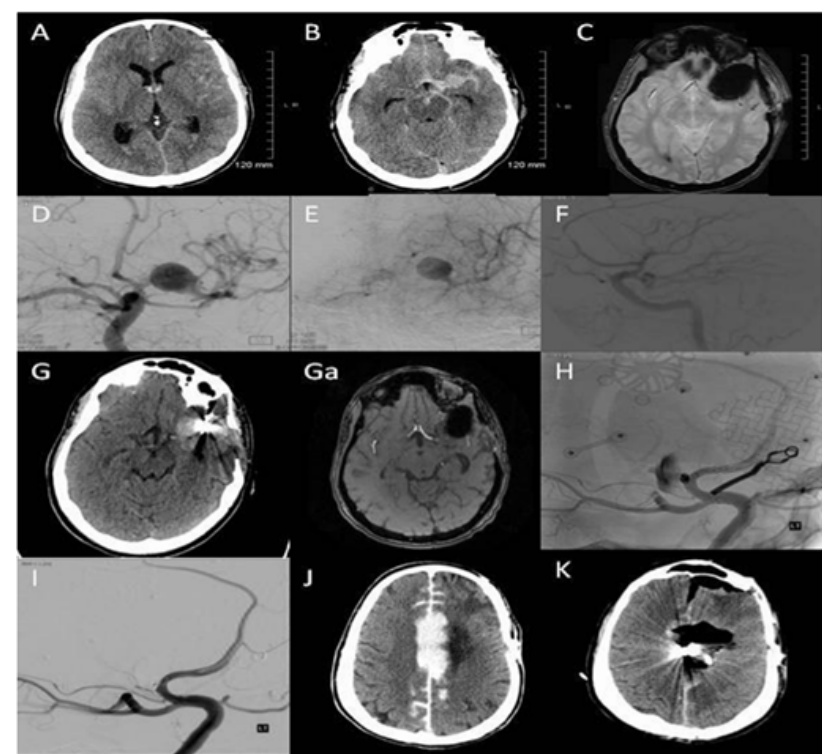

Figure $2 \mathrm{~A}-\mathrm{C}$ ) Admission CT-scan and MRI revealing a Fisher grade IV SAH related to a $15 \mathrm{~mm}$, ruptured, distal $\mathrm{MI}$ segment fusiform aneurysm; D,E) Angiogram demonstrating the anatomical relationship of this lesion to the lateral lenticulostriate perforators, as well as a small, early anterior frontal branch, which arose directly from the body of the fusiform dilatation; F) Intraoperative angiogram confirming good filling of the distal circulation through the bypass and native collateral flow, and near complete occlusion of the fusiform lesion; G,Ga) Follow-up CT-scan and MRI imaging showing interval growth of the fusiform aneurysm despite adequate surgical intervention; $\mathrm{H}, \mathrm{I})$ angiography showing active contrast extravasation related to a distal A3 iatrogenic perforation during stent deployment; J,K) post procedure CT-scan revealing an interhemispheric intraparenchymal hematoma which required emergency craniotomy.

Despite these interventions, the aneurysm continued to enlarge on subsequent follow-up imaging (Figure $2 \mathrm{G}$ and $\mathrm{Ga}$ ), and stent assisted coil embolization was recommended. Under general anesthesia and with a fully heparinized patient, standard endovascular technique was performed to deliver a Neuroform stent [Boston Scientific/ Target, Fremont, CA] $(4 \mathrm{~mm} \times 20 \mathrm{~mm})$ in a coaxial fashion over a microguidewire and deployed within the left A1 segment, back into the supraclinoid left ICA, across the neck or origin of the M1 segment aneurysm. Following successful stent deployment, diagnostic angiography failed to demonstrate any complication. This was followed by sub-selective catheterization of the distal ICA and proximal $2.5 \mathrm{~cm}$ MCA for deployment of a single DELTAPAQ [Micrus/ Endovascular, San Jose CA] 10 micro coil $(1.5 \mathrm{~mm}$ x $6 \mathrm{~cm})$. A diagnostic angiography run suggested appropriate coil placement at the proximal MCA trunk. The decision was then made to attempt placement of two additional stents across the aneurysm neck, to redirect flow and potentially occlude the lesion. Initially, a second $(4.5 \mathrm{~mm} \times 20 \mathrm{~mm})$ stent was deployed from the A1 segment back into the mid supraclinoid left ICA without any complications. During deployment of the 3rd stent, an iatrogenic guidewire perforation of the A3 segment took place. Active contrast extravasations necessitated an urgent endovascular occlusion of the left A3 segment (Figure 2H and 2I). Post procedure imaging revealed a large frontal intracranial hemorrhage, for which he underwent evacuation followed by decompressive craniectomy with areas of acute infarction in the paramedian frontal and parietal lobes (Figure 2J and $2 \mathrm{~K}$ ). Post-operatively, the patient remained in poor condition, and the family elected to withdraw support.

Case 3: A 53-year-old male active smoker, with a medical history of psychiatric symptoms, hypertension and dyslipidemia, was 
incidentally found to have a $14 \mathrm{~mm}$ right proximal M1 trunk dissecting MCA aneurysm (Figure 3A). Diagnostic angiography demonstrated several lenticulostriatal perforators' vessels arising from the dome of the fusiform dilatation. There was also an early anterior temporal cortical branch of moderate caliber which arose from the dilatation antero-inferior aspect (Figure 3B). The relationship of the aneurysm to these vessels was considered a contraindication to endovascular treatment.

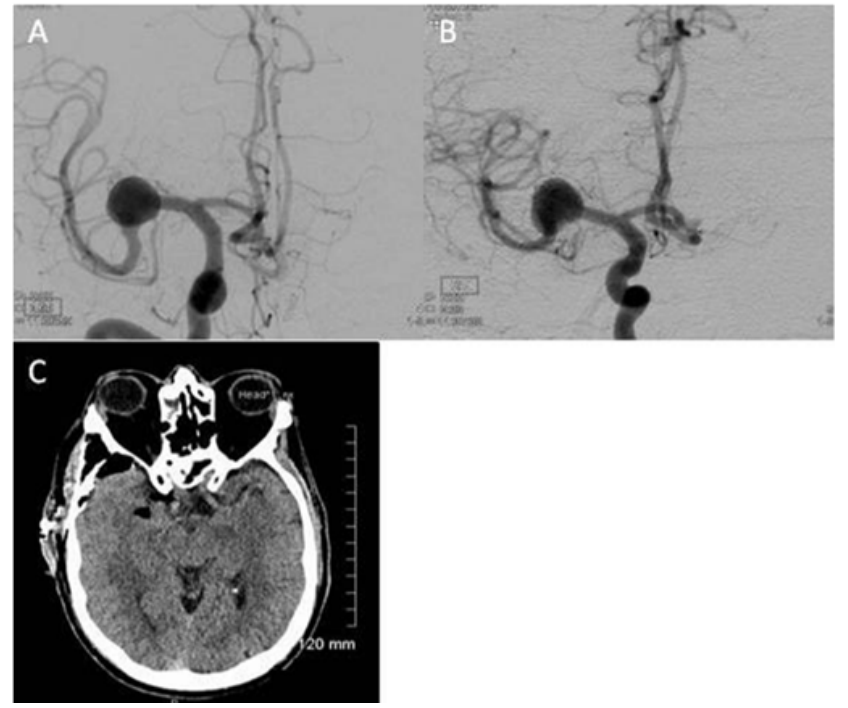

Figure $3 \mathrm{~A}, \mathrm{~B})$ Pre operative angiogram demonstrating an incidentally found $14 \mathrm{~mm}$ right proximal $\mathrm{MI}$ trunk dissecting MCA aneurysm. Diagnostic angiography also revealed several lenticulostriate perforators' vessels arising from the dome of the fusiform dilatation. In addition, there was an early anterior temporal cortical branch of moderate caliber which arose from the dilatation antero-inferior aspect; C) CT-scan showing post-procedure typical interval changes without any evidence of acute ischemia.

\section{Operative description}

The patient underwent microsurgical exploration with wrapping of his dissecting aneurysm. Intra-operatively, the aneurysm was found to be tucked below the insula. On inspection, the wall was white and atherosclerotic in appearance. The lenticulostriatal perforators arose from the superior aspect of the lesion as well as the aneurysm base. Due to the complex placement of the lenticulostriatal perforators clipping was deemed unfeasible. Since the patient was asymptomatic and not suffering from either hemorrhage or mass effect, a decision was made to wrap the aneurysm with Gore-Tex (Gore-SM) circumferentially, to decrease the risk of future expansion or bleeding. Post-operatively the patient experienced some gait disturbance felt to be related to his pharmacological regimen since radiological investigation failed to reveal evidence of acute or sub acute ischemia (Figure 3C). He was transferred to a transitional care unit in stable condition.

\section{Conclusion}

MCA-M1 dissecting aneurysms are best handled by a subspecialized neurovascular team working in a high volume center. Each patient must be managed individually with careful attention given to the risks and benefits of available treatment options. The most complex lesions, particularly those that are ruptured, may require multimodality, staged procedures. Despite seemingly good initial angiographic results, these lesions require close follow-up monitoring; aneurysms that appear to be adequately treated have been known to demonstrate regrowth and even re bleeding over a brief follow-up period.
As a result, early radiological follow-up is recommended in these cases as unexpected and asymptomatic lesion regrowth appears common and may portend impending re bleeding.

\section{Acknowledgments}

None.

\section{Conflicts of interest}

None.

\section{References}

1. Day AL, Gaposchkin CG, Yu CJ, et al. Spontaneous fusiform middle cerebral artery aneurysms: characteristics and a proposed mechanism of formation. J Neurosurg. 2003;99(2):228-240.

2. Fodstad H, Liliequist B, Wirell S, et al. Giant serpentine intracranial aneurysm after carotid ligation. Case report. $J$ Neurosurg. 1978;49(6):903-909.

3. Kitano I, Fukui M, Kinoshita K. Infantile cerebral aneurysm. Surg Neurol. 1981;16(5):386-390.

4. Sasaki O, Koike T, Takeuchi S, et al. Serial angiography in a spontaneous dissecting anterior cerebral artery aneurysm. Surg Neurol. 1991;36(1):49-53.

5. Yonas H, Agamanolis D, Takaoka Y, et al. Dissecting intracranial aneurysms. Surg Neurol. 1977;8(6):407-415.

6. Bederson JB, Spetzler RF. Anastomosis of the anterior temporal artery to a secondary trunk of the middle cerebral artery for treatment of a giant M1 segment aneurysm. Case report. J Neurosurg. 199276(5):863-866.

7. Yasagrill MG. Clinical Considerations, surgery of the intracranial aneurysms and results In: MG Y (Ed.), Micro Neuro Surgery, Volume 2, Georg Thieme Verlag, New York. 1984;pp.124-164.

8. Drake CG, Peerless SJ. Giant fusiform intracranial aneurysms: review of 120 patients treated surgically from 1965 to 1992 . J Neurosurg. 1997;87(2):141-162.

9. Nakatomi H, Segawa H, Kurata A, et al. Clinicopathological study of intracranial fusiform and dolichoectatic aneurysms: insight on the mechanism of growth. Stroke. 2000;31(4):896-900.

10. Ohkuma H, Suzuki S, Shimamura N, et al. Dissecting aneurysms of the middle cerebral artery: neuroradiological and clinical features. Neuroradiology. 2003;45(3):143-148.

11. Barbour PJ, Castaldo JE, Rae-Grant AD, et al. Internal carotid artery redundancy is significantly associated with dissection. Stroke. 199425(6):1201-1206.

12. Schievink WI, Prakash UB, Piepgras DG, et al. Alpha 1-antitrypsin deficiency in intracranial aneurysms and cervical artery dissection. Lancet. 1994343(8895):452-453.

13. Schievink WI, Puumala MR, Meyer FB, et al. Giant intracranial aneurysm and fibromuscular dysplasia in an adolescent with alpha 1antitrypsin deficiency. J Neurosurg. 199685(3):503-506.

14. Yamashita M, Tanaka K, Matsuo T, et al. Cerebral dissecting aneurysms in patients with moyamoya disease. Report of two cases. J Neurosurg. 1983;58(1):120-125.

15. Zhang QJ, Kobayashi S, Gibo $\mathrm{H}$, et al. Vertebrobasilar junction fenestration associated with dissecting aneurysm of intracranial vertebral artery. Stroke. 1994;25(6):1273-1275.

16. Greene KA, Anson JA, Spetzler RF. Giant serpentine middle cerebral artery aneurysm treated by extracranial-intracranial bypass. Case report. J Neurosurg. 1993;78(6):974-978. 
17. Hrbac T, Drabek P, Klement P, et al. A combined approach to treatment of the dissecting middle cerebral artery fusiform aneurysm. A case report. Interv Neuroradiol. 2009;15(3):349-354.

18. Spetzler RF, Schuster H, Roski RA (1980) Elective extracranialintracranial arterial bypass in the treatment of inoperable giant aneurysms of the internal carotid artery. J Neurosurg. 2009;53(1):22-27.

19. Spetzler RF, Carter LP. Revascularization and aneurysm surgery: current status. Neurosurgery. 1985;16(1):111-116.
20. Sundt TM Jr, Marsh WR. Bypass vein grafts for giant aneurysms and severe intracranial occlusive disease in the anterior and posterior circulation. In: Jr ST (Ed.), Occlusive cerebrovascular disease. Diagnosis and Surgical Management. WB Saunders, Philadelphia, pp. $1987 ; 439-464$.

21. Nussbaum ES, Madison MT, Myers ME, et al. Dissecting aneurysms of the posterior inferior cerebellar artery: retrospective evaluation of management and extended follow-up review in 6 patients. J Neurosurg. 2008;109(1):23-27. 